\title{
EFEITO DA EXPLORAÇÃO FLORESTAL SELETIVA EM UMA FLORESTA ESTACIONAL SEMIDECIDUAL ${ }^{1}$
}

\author{
Sueli Sato Martins ${ }^{2}$, Laércio Couto ${ }^{3}$, Carlos Cardoso Machado ${ }^{3}$ e Agostinho Lopes de Souza ${ }^{3}$
}

\begin{abstract}
RESUMO - O objetivo deste trabalho foi avaliar a capacidade de sustentação de uma floresta nativa explorada, mediante o conhecimento da estrutura da floresta antes e após a exploração florestal, buscando recomendar medidas para minimizar os impactos causados pelas atividades de exploração sobre a vegetação adulta remanescente. Observou-se aumento no número de espécies e famílias dois anos após a exploração, em nível das adultas, ou seja, $\mathrm{DAP} \geq 5 \mathrm{~cm}$, em razão do ingresso de espécies, o mesmo não sendo observado para classes menores que esta. O padrão de distribuição das espécies foi bastante afetado pela exploração, mas devido ao dinamismo da floresta ele deverá ser avaliado na época da próxima exploração.
\end{abstract}

Palavras-chave: Exploração seletiva, composição florística e Floresta Estacional Semidecidual.

\section{EFFECTS OF SELECTIVE LOGGING ON A SEMIDECIDUOUS SEASONAL FOREST}

\begin{abstract}
The objective of this study was to evaluate the sustainability capacity of a logging native forest based on the knowledge of its structure before and after logging, aiming to determine measures to minimize the impacts caused by harvesting on the remaining vegetation. It was observed an increase in the number of families and species two years after logging at the adult level, i.e., $D B H \geq 5 \mathrm{~cm}$, due to ingrowth, the same not being observed in the lowest level. The distribution pattern was affected by logging but due to the dynamism of the forest, it should be evaluated before the next logging.
\end{abstract}

Key words: $\quad$ Selective logging, floristic composition and Semidecidual Seasonal Forest.

\section{INTRODUÇÃO}

Os impactos da exploração madeireira nas florestas nativas, considerando os efeitos na vegetação adulta remanescente, na regeneração natural e no solo, devem ser cuidadosamente observados no manejo dessas florestas. Tais impactos têm implicações diretas na escolha do sistema de manejo a ser aplicado e na busca de respostas a questões básicas relacionadas com a auto-ecologia das espécies envolvidas.

A questão fundamental é que essas florestas devem gerar produtos para novas colheitas, princípio básico do rendimento sustentável, sendo a regeneração natural a condição vital que permite a sua conservação e preservação. Assim, a colheita das florestas nativas deve considerar o conceito de sustentabilidade, uma vez que novos ciclos de corte devem ser realizados.

O manejo das florestas nativas deve considerar o desenvolvimento de sistemas que garantam o crescimento das árvores e de produtos desejáveis, como a conservação dos recursos hídricos e florísticos, que têm sido bastante degradados pela crescente pressão antrópica sobre eles (Good et al., 1993).

O manejo sustentável das florestas naturais é alcançado quando os responsáveis pelas atividades de colheita consideram, além da biodiversidade, o aspecto social da

1 Recebido para publicação em 19.9.2001.

Aceito para publicação em 19.2.2003.

2 Eng. Florestal, Universidade Estadual de Maringá, 87020-900 Maringá-PR, <ssmartins@uem.br>. ${ }^{3}$ Professores do Dep. de Engenharia Florestal da Universidade Federal de Viçosa - UFV, 36570-000 Viçosa-MG. 
floresta e as práticas de regeneração (Adlard, 1993; Lee, 1993).

Os danos causados pela colheita florestal devem ser cuidadosamente investigados, para detectar seus efeitos sobre a floresta remanescente, na tentativa de eliminálos ou reduzi-los ao mínimo, evitando prejuízo às produções futuras. Assim, apesar de haver regeneração natural após a colheita, essa nem sempre é adequada ao rendimento futuro dessa floresta.

Os objetivos deste trabalho foram:

a) avaliar a capacidade de sustentação de uma Floresta Estacional Semidecidual no sudoeste do Paraná;

b) conhecer a estrutura e a composição florística da floresta, antes e após a exploração florestal em uma floresta nativa; $\mathrm{e}$

c) recomendar medidas que minimizem os impactos causados pelas atividades de exploração florestal na vegetação remanescente e na regeneração natural.

\section{MATERIAL E MÉTODOS}

\subsection{Caracterização da Área de Estudo}

A área em estudo localiza-se no município de Quedas do Iguaçu, Paraná, nas coordenadas 52'54'39'W e $25^{\circ} 27^{\prime} 22^{\prime \prime}$, na bacia hidrográfica do rio Iguaçu, de propriedade da Araupel S.A. A região está enquadrada no mapa fitogeográfico do Estado do Paraná como Floresta Estacional Semidecidual (IBGE, 1992; Roderjan et al., 1992). Essa região é condicionada pela dupla estacionalidade climática, uma tropical, com época de chuvas de verão seguida por estiagem acentuada, e outra subtropical, sem período seco, mas com seca fisiológica provocada pelas baixas temperaturas de inverno, que são inferiores a $15{ }^{\circ} \mathrm{C}$. O clima é classificado como $\mathrm{Cfa}$, segundo Köppen, subtropical úmido, com verões quentes e temperatura média anual de $20^{\circ} \mathrm{C}$, superior a $22^{\circ} \mathrm{C}$ no mês mais quente e inferior a $18^{\circ} \mathrm{C}$ no mês mais frio, e com invernos com menos de três geadas, anualmente. A precipitação média anual é de $1.780 \mathrm{~mm}$ e a do mês mais seco, de $60 \mathrm{~mm}$ (EMBRAPA, 1984).

O relevo é suave-ondulado, com vertentes longas e altitude de 450 a $600 \mathrm{~m}$ acima do nível do mar. O tipo de solo é Latossolo Vermelho Distroférrico, com presença de solos litólicos (EMBRAPA, 1999).

R. Árvore, Viçosa-MG, v.27, n.1, p.65-70, 2003

\subsection{Amostragem e Coleta dos Dados}

Amostrou-se uma área de 398 ha, antes da exploração florestal. O inventário foi realizado, utilizando-se o método de amostragem sistemática. Para isto, traçaramse sobre o mapa da área linhas no sentido leste-oeste, nas quais foram alocadas 19 parcelas de 0,1 ha, tendo sido amostrado $1 \%$ da área total. Cada parcela de $20 \times 50 \mathrm{~m}$ foi subdividida em subparcelas de $20 \times 10 \mathrm{~m}$, nas quais foram medidos os indivíduos arbóreos com DAP $\geq 5 \mathrm{~cm}$, anotando-se as seguintes características: nome vulgar regional das espécies lenhosas; diâmetro a 1,30 m do solo; altura comercial de todos os indivíduos; altura total; sanidade das árvores; e presença de cipós na árvore.

\subsection{Identificação do Material Botânico}

A identificação do material botânico foi realizada com o auxílio de mateiros, familiarizados com a flora da região, que forneceram o nome vulgar regional das espécies. O material fértil (flores e, ou, frutos) foi coletado, para identificação taxonômica, por especialistas em sistemática vegetal. O material classificado como nãoidentificado neste trabalho foi devido à não-obtenção de material fértil durante o transcurso do trabalho, por causa da periodicidade de floração e frutificação que ocorre com as espécies florestais.

\subsection{Análise Estrutural da Floresta}

\section{4. 1. Parâmetros Florísticos}

O índice de diversidade de Shannon e Weaver (H') foi calculado com base no número de indivíduos de cada espécie e no total de indivíduos (Martins, 1979), e embora seja influenciado pela amostragem este índice fornece uma boa indicação da diversidade de espécies.

\subsubsection{Parâmetros Fitossociológicos}

A análise dos parâmetros fitossociológicos envolveu estimativas da estrutura horizontal. A estrutura horizontal refere-se à distribuição espacial das espécies arbóreas que compõem a população, tendo sido utilizados a densidade, a freqüência e o índice do valor de importância.

\subsection{Avaliação dos Efeitos da Exploração Florestal sobre a Estrutura da Floresta}

Para avaliação dos efeitos da exploração sobre a vegetação remanescente, foram utilizadas sete parcelas, 
envolvendo a área colhida, que haviam sido inventariadas antes e após a exploração, tendo sido avaliados os seus efeitos sobre a composição florística e sobre a estrutura horizontal. O tamanho das parcelas foi de 20x50 m, subdividida em subparcelas de 20x10 m.

Para avaliar os efeitos da exploração sobre a estrutura da floresta, as espécies foram classificadas em três categorias: comerciais, que são aquelas espécies comercializadas pela empresa; potenciais, aquelas que alcançaram $40 \mathrm{~cm}$ de DAP, empregadas na região; e outras que não são comercializadas.

O sistema de exploração obedeceu àquele autorizado pela legislação florestal vigente na época, ou seja, poderiam ser exploradas $70 \%$ das árvores com DAP acima de $40 \mathrm{~cm}$.

\section{RESULTADOS E DISCUSSÃO}

\subsection{Efeitos da Exploração Florestal sobre a Composição Florística}

Pela amostragem realizada, considerando somente a fração colhida, observaram-se, antes da primeira exploração, 271 indivíduos, pertencentes a 19 famílias e 25 espécies, no primeiro nível de abordagem (DAP $>5 \mathrm{~cm}$ ), e 241 indivíduos, pertencentes a 24 famílias e 38 espécies, no segundo nível de abordagem (DAP $\leq 5 \mathrm{~cm}$ ). Após a exploração observaram-se, no primeiro nível de abordagem, 164 indivíduos, pertencentes a 21 famílias e 34 espécies, e 135 indivíduos, pertencentes a 16 famílias e 27 espécies, no segundo nível de abordagem (Quadro 1).

Quadro 1 - Florística na área da Charqueada, antes e após a exploração

Table 1 - Charqueada area floristic composition before and after logging

\begin{tabular}{|l|c|c|c|}
\hline $\begin{array}{c}\text { Época de } \\
\text { Exploração/ } \\
\text { Classe de } \\
\text { Diâmetro }\end{array}$ & $\begin{array}{c}\text { Número de } \\
\text { Indivíduos }\end{array}$ & $\begin{array}{c}\text { Número de } \\
\text { Famílias }\end{array}$ & $\begin{array}{c}\text { Número de } \\
\text { Espécies }\end{array}$ \\
\hline \multicolumn{4}{|c|}{ Antes da Exploração } \\
\hline DAP $\geq 5 \mathrm{~cm}$ & 271 & 19 & 25 \\
$\mathrm{DAP} \leq 5 \mathrm{~cm}$ & 241 & 24 & 38 \\
\hline \multicolumn{4}{|c|}{ Após a Exploração } \\
\hline $\mathrm{DAP} \geq 5 \mathrm{~cm}$ & 164 & 21 & 34 \\
$\mathrm{DAP} \leq 5 \mathrm{~cm}$ & 135 & 16 & 27 \\
\hline
\end{tabular}

Pode-se observar que, após a exploração, houve aumento no número de espécies e de famílias no primeiro nível de abordagem, ou seja, DAP $\geq 5 \mathrm{~cm}$, em razão do ingresso ocorrido entre o período da primeira amostragem e a exploração, realizada dois anos após. O mesmo não ocorreu em relação às espécies do segundo nível (DAP $<5 \mathrm{~cm}$ ), quando se verificou redução de 38 para 27 espécies em regeneração.

O índice de diversidade de Shannon e Weaver (H'), considerando indivíduos a partir de $10 \mathrm{~cm}$ de altura, foi de 2,29, antes da exploração, e de 2,60, após a exploração. Esperava-se que ocorresse aumento na diversidade após um distúrbio na área, em razão da abertura de clareiras, ocasionado pelo espaço de tempo ocorrido entre a primeira amostragem e a exploração, realizada dois anos após.

\subsection{Efeitos da Exploração Florestal sobre o Padrão de Agregação das Espécies}

O padrão de agregação das espécies foi muito afetado pela atividade de exploração (Quadro 2), observado em algumas espécies comercializáveis, como Apuleia leiocarpa (grápia), Luehea divaricata (açoitacavalo), Nectandra lanceolata (canela- amarela) e Cedrela fissilis (cedro-rosa), que apresentavam padrão agregado ou tendência à agregação antes da exploração, passando ao padrão aleatório após a exploração. A mudança no padrão de distribuição espacial das espécies também foi observado por Kammescheidt et al. (2001), na colheita de florestas na Venezuela. As espécies que ingressaram na área após a exploração, em virtude de sua baixa freqüência, foram as que apresentaram padrão aleatório, aumentando de 12 para $26 \%$ as espécies com

Quadro 2 - Padrão de distribuição espacial das espécies arbóreas na Charqueada, antes e após a exploração, de acordo com o índice de Mcguinnes (DAP $\geq 5 \mathrm{~cm}$ )

Table 2 - Spatial distribution pattern of the trees, in Charqueada, before and after logging, considering Mcguinnes index $(D B H \geq 5 \mathrm{~cm})$

\begin{tabular}{|l|c|c|c|}
\hline \multirow{2}{*}{$\begin{array}{c}\text { Época de } \\
\text { Exploração }\end{array}$} & \multicolumn{3}{|c|}{ Padrão de Agregação } \\
\cline { 2 - 4 } & Agregado & $\begin{array}{c}\text { Tendência à } \\
\text { Agregação }\end{array}$ & Aleatório \\
\hline Antes & $32(8)^{*}$ & $56(14)^{*}$ & $12(3)^{*}$ \\
Após & $12(4)^{*}$ & $26(9)^{*}$ & $62(21)^{*}$ \\
\hline
\end{tabular}

* Número de espécies. 
esse padrão de distribuição. O processo de sucessão da floresta é muito dinâmico, sendo necessário um período de tempo bastante longo para chegar às conclusões sobre o padrão de distribuição das espécies (Carvalho, 1992).

Uma vez que o padrão de distribuição espacial das espécies é afetado pelas variações nas condições locais, essas mudanças de comportamento tendem a ocorrer de acordo com a evolução dos estádios de sucessão florestal (Matteucci \& Colma, 1982). O conhecimento do padrão de distribuição das espécies é fundamental na tomada de decisão sobre o manejo silvicultural a ser aplicado na área. No caso de espécies com padrão agregado, devemse manter alguns indivíduos de tamanho comercial, para que continue a ocorrer a distribuição natural. Já para aquelas com distribuição aleatória, devem-se manter indivíduos para fornecimento de novos cortes e, principalmente, para garantir a perpetuidade da espécie na área.

\subsection{Efeitos da Exploração sobre a Estrutura da Floresta da Charqueada}

\subsubsection{Estrutura Horizontal}

Apesar do aumento no número de espécies por meio de seu ingresso na área, observou-se redução no número de indivíduos após a intervenção (Quadro 3). Com este resultado, fica claro que a floresta deverá ser manejada, para que novo estoque madeireiro possa ser colhido.

Das 25 espécies presentes antes da exploração, dez pertencem à classe das comerciais; cinco à das potenciais; e dez a das de outros usos. No entanto, é visível a dominância das espécies comerciais na área, com $45 \%$ do valor de importância.

Após a exploração, a dominância passou para o grupo das outras espécies, com $40 \%$ do valor de importância, e as espécies comerciais passaram para 33\%, demonstrando a redução no valor comercial dessa floresta.

\subsubsection{Estoque de Crescimento}

O efeito da exploração sobre o estoque de crescimento $(5 \leq \mathrm{DAP}<40 \mathrm{~cm}$ ) pode ser percebido pelo número de árvores por hectare, distribuído por classes de tamanho e por categoria de espécies (Quadro 4). Verifica-se que houve aumento no número de árvores na

Quadro 3 - Distribuição do número de indivíduos, por categoria de espécies, na área da Charqueada, antes e após a exploração

Table 3 - Distribution of number individuals, by species, at "Charqueada" before and after selective logging

\begin{tabular}{|c|c|c|c|c|}
\hline \multirow{2}{*}{$\begin{array}{c}\text { Grupo de } \\
\text { Espécies }\end{array}$} & \multicolumn{4}{|c|}{ Época de Exploração } \\
\cline { 2 - 5 } & Antes & \multicolumn{2}{c|}{ Depois } \\
\cline { 2 - 5 } & $\begin{array}{c}\text { Número de } \\
\text { Indivíduos }\end{array}$ & $\begin{array}{c}\text { IVI } \\
(\%)\end{array}$ & $\begin{array}{c}\text { Número de } \\
\text { Indivíduos }\end{array}$ & $\begin{array}{c}\text { IVI } \\
(\%)\end{array}$ \\
\hline Comerciais & 88 & 45 & 47 & 33 \\
Potenciais & 56 & 27 & 35 & 27 \\
Outras & 127 & 28 & 82 & 40 \\
\hline \multicolumn{1}{|c|}{ Total } & 271 & 100 & 164 & 100 \\
\hline
\end{tabular}

Quadro 4 - Estimativas médias do número de árvores por hectare na área da Charqueada antes e após a exploração, para as espécies comerciais, potenciais e outras

Table 4-Number of trees / ha, before and after logging, at "Charqueada" for commercial, potential and other species

\begin{tabular}{|c|c|c|c|c|c|c|c|c|c|c|c|c|}
\hline \multirow{2}{*}{ Espécies } & \multirow{2}{*}{ Período } & \multicolumn{11}{|c|}{ Classe de DAP } \\
\hline & & 7,5 & 17,5 & 27,5 & 37,5 & 47,5 & 57,5 & 67,5 & 77,5 & 97,5 & 117,5 & Total \\
\hline \multirow{2}{*}{ Comerciais } & A & 15,73 & 34,29 & 11,44 & 22,86 & 5,72 & 15,72 & 8,57 & 2,86 & 5,71 & 2,86 & 125,76 \\
\hline & $\mathrm{D}$ & 37,16 & 11,43 & 1,43 & 10,01 & - & 5,72 & 1,43 & - & - & - & 67,18 \\
\hline \multirow{2}{*}{ Potenciais } & A & 11,43 & 17,14 & 5,72 & 20,00 & 11,44 & 5,72 & 5,72 & 2,86 & - & - & 60,03 \\
\hline & $\mathrm{D}$ & 15,73 & 4,29 & 1,43 & 10,00 & 8,57 & - & 4,29 & - & - & - & 32,87 \\
\hline \multirow{2}{*}{ Outras } & A & 22,86 & 104,29 & 48,57 & 5,72 & - & - & - & - & - & - & 201,44 \\
\hline & $\mathrm{D}$ & 42,88 & 55,42 & 24,29 & - & - & - & - & - & - & - & 134,33 \\
\hline \multirow{2}{*}{ Total } & A & 50,22 & 155,72 & 65,73 & 48,58 & 17,16 & 21,44 & 14,29 & 5,71 & 5,71 & 2,86 & 387,22 \\
\hline & $\mathrm{D}$ & 95,77 & 71,44 & 27,15 & 20,01 & 8,57 & 5,72 & 5,72 & - & - & - & 234,38 \\
\hline
\end{tabular}

R. Árvore, Viçosa-MG, v.27, n.1, p.65-70, 2003 
Quadro 5 - Estimativas médias do volume por hectare, antes e após a exploração na área da Charqueada, por classes de DAP, para as espécies comerciais, potenciais e outras

Table 5 - Estimates of Volume/ha, before and after logging at "Charqueada" by DBH classes for the commercial, potential and other species

\begin{tabular}{|c|c|c|c|c|c|c|c|c|c|c|c|c|}
\hline \multirow{2}{*}{ Espécies } & \multirow{2}{*}{ Período } & \multicolumn{11}{|c|}{ Classe de DAP } \\
\hline & & 7,5 & 17,5 & 27,5 & 37,5 & 47,5 & 57,5 & 67,5 & 77,5 & 97,5 & 117,5 & Total \\
\hline \multirow{2}{*}{ Comerciais } & A & 0,356 & 3,827 & 5,614 & 19,993 & 6,333 & 33,254 & 21,744 & 11,246 & 32,565 & 26,403 & 161,335 \\
\hline & $\mathrm{D}$ & 0,455 & 1,223 & 0,677 & 7,454 & - & 10,601 & 3,731 & - & - & - & 24,141 \\
\hline \multirow{2}{*}{ Potenciais } & A & 0,463 & 1,243 & 2,607 & 15,581 & 12,713 & 9,245 & 16,430 & 10,504 & - & - & 68,786 \\
\hline & $\mathrm{D}$ & 0,272 & 0,308 & 0,356 & 6,204 & 5,102 & - & 9,943 & - & - & - & 22,185 \\
\hline \multirow{2}{*}{ Outras } & A & 0,202 & - & - & 3,103 & - & - & - & - & - & - & 3,305 \\
\hline & $\mathrm{D}$ & 0,192 & 0,327 & - & - & - & - & - & - & - & - & 0,519 \\
\hline \multirow{2}{*}{ Total } & A & 1,021 & 5,070 & 8,221 & 38,677 & 19,046 & 42,499 & 38,174 & 21,750 & 32,565 & 26,403 & 233,426 \\
\hline & $\mathrm{D}$ & 0,919 & 1,858 & 1,033 & 13,658 & 5,102 & 10,601 & 13,674 & - & - & - & 46,845 \\
\hline
\end{tabular}

primeira classe de tamanho, em razão do ingresso destas na área, uma vez que a primeira amostragem foi realizada dois anos antes da exploração.

Algumas espécies, como Campomanesiaxanthocarpa, Xylosma warburgii, Myrocarpus frondosus, Jacaranda micrantha, Covatã, Sapium glandulatum e Inga striata, devem fazer parte do ingresso na área, em virtude do baixo número de árvores na primeira classe de DAP.

Pode-se observar que houve ingresso das árvores na categoria das espécies comerciais, na primeira classe de DAP (Quadro 5). A partir desta classe houve redução no volume para as demais classes, tendo sido retirados todos os indivíduos do estoque de exploração e mantidas apenas três das espécies da categoria das comerciais, a saber: Lonchocarpus muelhbergianus (rabo-de-bugio), Patagonula americana (guajuvira) e Luehea divaricata (açoita-cavalo), esta última somente no estoque de exploração, e não no estoque de crescimento.

Foram também mantidos indivíduos de duas espécies potenciais no estoque de exploração, a saber: Machaerium brasiliensis (sapuva) e Matayba elaeagnoides (miguel-pintado).

\section{CONCLUSÕES}

O índice de McGuiness indicou que, antes da exploração, a maioria das espécies apresentou tendência à agregação, seguida pelo padrão agregado e, com menor freqüência, pelo padrão aleatório. É comum a ocorrência deste fato em florestas tropicais, onde espécies mais abundantes ocorrem agrupadas ou com tendência ao agrupamento. Após a exploração, a maioria passou para o padrão aleatório, em razão da retirada de espécies comerciais da área colhida.

O índice de diversidade de Shannon e Weaver (H'), considerando indivíduos a partir de $10 \mathrm{~cm}$ de altura, foi de 2,29 e 2,60, antes e após a exploração, respectivamente. Esse aumento na diversidade pode ser devido ao ingresso de espécies na área, bem como ao próprio distúrbio da exploração e à abertura de clareiras na floresta.

O estoque de crescimento da área explorada foi mantido para a maioria das espécies observadas, sem, no entanto, ter havido a preocupação de manter adequadamente esse estoque, uma vez que não há planejamento de sua extração.

Uma vez que o processo é extremamente complexo, deve-se continuar o levantamento dessas espécies, procurando observar os fatores responsáveis pela continuidade da comunidade.

As espécies que se encontravam bem distribuídas em todas as classes de diâmetro acima de $5 \mathrm{~cm}$, como Ocotea catharinensis e Lonchocarpus muehlbergianus, não sofreram pressão de seleção, sendo observados indivíduos após a exploração.

Espécies como Cedrela fissilis, do grupo das comerciais, podem ser consideradas como ideais, ou seja, com alta regeneração natural e resistente aos impactos da

R. Árvore, Viçosa-MG, v.27, n.1, p.65-70, 2003 
exploração, uma vez que foram observadas nas classes $\mathrm{U}$ (menor que $30 \mathrm{~cm}$ ) e com DAP maior que $5 \mathrm{~cm}$.

\section{REFERÊNCIAS BIBLIOGRÁFICAS}

ADLARD,, P. G. Sustainability. The concept of sustainability as applied to tree planting. Oxford: SHELL/ WWF. Tree Plantation Review, 1993. 31 p. (Study, 5)

CARVALHO, J. O. P. Structure and dynamics of a logged over Brazilian Amazonian rain forest. 1992. 215 f. Tese (Ph.D.) - Oxford University, Oxford, 1992.

EMPRESA BRASILEIRA DE PESQUISA AGROPECUÁRIA - EMBRAPA. Serviço Nacional de Levantamento e Conservação de Solos. Levantamento de reconhecimento dos solos do estado do Paraná. Curitiba: 1984. $791 \mathrm{p}$.

EMPRESA BRASILEIRA DE PESQUISA NA AGROPECUÁRIA - EMBRAPA. Sistema Brasileiro de Classificação de Solos. Rio de Janeiro: 1999. 180 p.

INSTITUTO BRASILEIRO DE GEOGRAFIA E ESTATÍSTICA - IBGE. Manual técnico da vegetação brasileira. Rio de Janeiro: 1992. 92 p.
GOOD, J.; LAWSON, G.; STEVENS, P. Natural environment. Bangor: SHELL/WWF. Tree Plantation Review, 1993. 134 p. (Study, 8)

KAMMESCHEIDT, L.; LEZAMA, A. T.; FRANCO, W.; PLONCZAK, M. History of logging and silvicultural treatments in the western Venezuelan plain forests and the prospect for sustainable forest management. Forest Ecology and Management, v. 148, p. 1-20, 2001.

LEE, S. A. Biodiversity. Manchester: SHELL/WWF. Tree Plantation Review, 1993. 34 p. (Study, 6)

MARTINS, F. R. O método de quadrantes e a fitossociologia de uma floresta residual do interior do Estado de São Paulo: Parque Estadual de Vassununga. 1979. 239 f. Tese (Doutorado em em Biociências) Universidade de São Paulo, São Paulo, 1979.

MATTEUCCI, S. D.; COLMA, A. Metodologia para el estudio de la vegetación. Washington, Secretaria General de la Organización de los Estados Americanos, 1982. 168 p. (Monografia, 22).

RODERJAN, C. V.; KUNIYOSHI, Y. S.; GALVÃO, F. As regiões fitogeográficas do Estado do Paraná. Acta Forestalia Brasiliensis, v. 1, n. 19, p. 3, 1992. 